\title{
Norois
}

Environnement, aménagement, société

$236 \mid 2015$

Présence chinoise en Arctique, nautisme, ville-port, vulnérabilité, inondation

\section{Vers un nouveau modèle de la plaisance ? Profils et pratiques des plaisanciers du Finistère}

Towards a new model of yachting? Recreational boater's profiles and practices in Finistère (NW France)

Lucien Perras, Ingrid Peuziat, Iwan Le Berre, Adeline Maulpoix, Louis Brigand et Nicolas Le Corre

\section{OpenEdition}

\section{Journals}

\section{Édition électronique}

URL : https://journals.openedition.org/norois/5706

DOI : $10.4000 /$ norois.5706

ISBN : 978-2-7535-4945-6

ISSN : 1760-8546

Éditeur

Presses universitaires de Rennes

Édition imprimée

Date de publication : 30 décembre 2015

Pagination : 39-56

ISBN : 978-2-7535-4903-6

ISSN : 0029-182X

Référence électronique

Lucien Perras, Ingrid Peuziat, Iwan Le Berre, Adeline Maulpoix, Louis Brigand et Nicolas Le Corre,

«Vers un nouveau modèle de la plaisance? Profils et pratiques des plaisanciers du Finistère », Norois [En ligne], 236 | 2015, mis en ligne le 30 décembre 2017, consulté le 01 février 2022. URL : http:// journals.openedition.org/norois/5706 ; DOI : https://doi.org/10.4000/norois.5706 


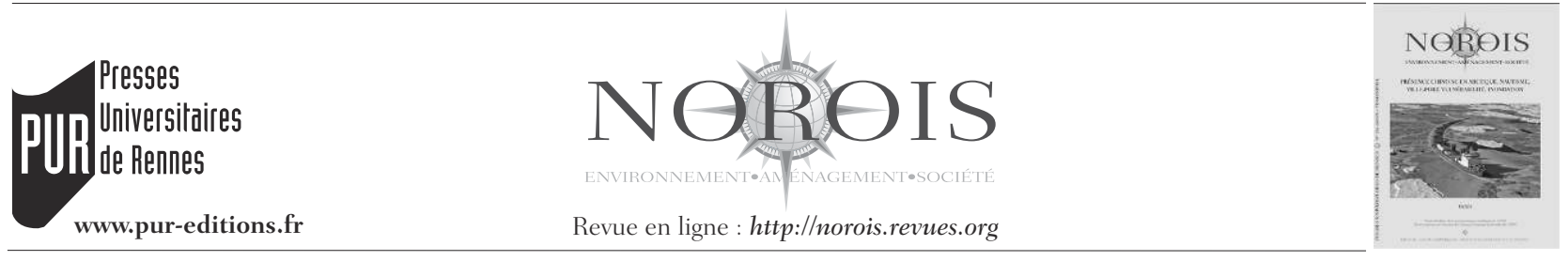

\title{
Vers un nouveau modèle de la plaisance? Profils et pratiques des plaisanciers du Finistère
}

\author{
Towards a New Model of Yachting? \\ Recreational Boater's Profiles and Practices in Finistère (NW France)
}

\section{Lucien Perras ${ }^{\mathrm{a}}$, Ingrid Peuziat ${ }^{* a}$, Iwan Le Berre ${ }^{\mathrm{a}}$, Adeline MaulpoiX ${ }^{\mathrm{a}}$, Louis Brigand ${ }^{a}$ et Nicolas Le Corre ${ }^{a}$}

\footnotetext{
*Auteur correspondant LETG (ingrid.peuziat@univ-brest.fr)

a Laboratoire LETG- Brest Géomer - UMR 6554 CNRS (université de Bretagne Occidentale), Institut Universitaire

Européen de la Mer - Technopôle Brest-Iroise, Rue Dumont-D’Urville - 29280 Plouzané, France.
}

Résumé : La démocratisation de la plaisance à partir des années 1960, s’est traduite par un fort développement des infrastructures dédiées sur l'ensemble des côtes de France, ainsi que par la structuration d'une filière nautique importante d'un point de vue économique et territorial. Cependant, le ralentissement du nombre des nouvelles immatriculations de bateaux interpelle les gestionnaires et les acteurs économiques de cette filière. Pour bien appréhender les questions complexes que pose cette évolution, il est indispensable de caractériser les profils et les pratiques des plaisanciers.

Cet article présente les résultats d'une enquête en ligne, diffusée à l'échelle du département du Finistère entre 2012 et 2013. Les données recueillies auprès d'environ 1500 plaisanciers, mettent en évidence le vieillissement de la population pratiquant la plaisance, matérialisant ainsi son faible renouvellement. En effet, les plus jeunes apparaissent désormais attirés par de nouvelles pratiques (sports de glisse, location de voilier dans des destinations dépaysantes) en lien avec le développement de la mobilité individuelle. Cette évolution soulève par conséquent d'importantes questions relatives au devenir des infrastructures nautiques et à la nécessaire adaptation des services associés (location, co-navigation...) pour parvenir à attirer de nouveaux plaisanciers.

Abstract: The democratization of yachting in the 1960s, resulted in strong development of dedicated infrastructures all along the coasts of France, as well as in the structuration of a nautical sector of major economic and territorial importance. However, the slowdown in the number of new registrations of boats challenges managers and economic stakeholders in this sector. The characterization of boaters profiles and practices, is crucial to understand the complex issues raised by these evolutions.

This article presents the results of an online survey distributed across the Finistere department between 2012 and 2013. Data collected from around 1.500 boaters, highlight the aging of the boasters population and its low turnover. Indeed, younger boaters are now attracted to new practices (windsurf, surf, sailboat rentals in exotic destinations, etc.) in connection with the development of individual mobility. This raises important questions about the future of boating facilities and the necessary adaptation of related services (rental, co-browsing...) to attract new boaters.

Mots clés : nautisme - plaisance - enquête en ligne - Finistère - bassin de navigation

Keywords: recreational boating - yachting - on-line survey - Finistère - yachting area 


\section{INTRODUCTION}

Réservée auparavant à une élite sociale, la plaisance a connu à partir des années 1960 une démocratisation (Bernard, 1993; Lageiste, 1995; Widmer et Underwood; 2004, Peuziat, 2005). Cette époque est en effet marquée par une politique d'aménagement et d'équipement du littoral conséquente concrétisée notamment par la construction de nouveaux ports de plaisance. Parallèlement, le développement de nouveaux matériaux (composites notamment) et de processus de fabrication par sérialisation permet une baisse sensible du coût des bateaux (Bernard, 2000). Ainsi, le nombre de plaisanciers ${ }^{1}$ n'a cessé de crôitre : en France métropolitaine, toutes pratiques confondues, il est aujourd'hui estimé à plus de quatre millions tandis que le nombre de bateaux de plaisance immatriculés est évalué à 1005252 , dont 199949 à voile et 754139 à moteur (DGITM, 2013).

Néanmoins, depuis 2009 on observe un ralentissement du nombre des nouvelles immatriculations de bateaux (DGITM, 2013). S'agit-il d'une évolution conjoncturelle, liée à un contexte économique peu favorable au développement, voire au maintien d'une activité de loisir relativement onéreuse? Cette évolution traduit-elle le vieillissement de la population de plaisancier, posant ainsi la préoccupante question de son renouvellement (conseil général du Finistère, 2015) ? Ou bien est-elle simplement le reflet de la diversification de l'offre de loisirs et d'un changement de pratiques des usagers du nautisme (Bernard, 2005)? Cette évolution interpelle particulièrement les collectivités et les établissements en charge de la gestion des équipements et des aménagements liés à la plaisance. Les questions complexes qu'elle pose nécessitent en tous cas une meilleure connaissance des profils et des pratiques des plaisanciers (D’Aboville, 2013).

\section{Connaître les profils et les pratiques des plaisanciers}

Malgré l'importance économique et territoriale de la filière nautique en France, l'activité de plaisance de même que les caractéristiques de la population

1. Est considéré comme plaisancier une personne pratiquant une activité nautique de loisir réalisée à partir d'un bateau propulsé à la voile ou au moteur (Miossec, 2012). qui la pratique restent relativement mal connues. En premier lieu, il s'agit d'une activité qui comporte de nombreuses dimensions - par la diversité des usages, des comportements et des attentes - et qui intéresse par conséquent des chercheurs de disciplines diverses à l'origine d'un certain éparpillement des travaux (médecine, droit, sociologie, ethnologie, géographie etc.). De plus, diverses contraintes s'imposent aux chercheurs travaillant sur les espaces marins et littoraux, notamment en sciences humaines et sociales, pour l'analyse des usages maritimes non encadrés : dispersion de l'activité et des infrastructures liées, diversité des organismes d'encadrement, caractère souvent individualiste de l'activité (Peuziat et Le Berre, 2015).

Malgré ces contraintes, les recherches menées en France en sciences humaines et sociales, se sont intéressées relativement tôt à la navigation de plaisance : en ethnologie et sociologie sous l'angle du sport et de la compétition, des profils des pratiquants ou encore des représentations (Falt, 1981 ; Michot, 1998 ; Créac'h, 2003; Le Bars et Lacombe, 2011 ; Brulé-Josso, 2012); en géographie par la description des aménagements, des ports de plaisance et de leur rôle dans la structuration et la construction des territoires plaisanciers (Bernard 1993; Brigand et al., 2010 ; Clerc Giraudo, 2002; Desse, 2000; Lageiste 1994; Marrou, 2011; Retière, 2003; Richez, 1992; Sonnic, 2004; Tonini et Trouillet, 2005). Enfin, l'augmentation de l'activité parallèlement à la multiplication des usages en mer (loisirs, activités professionnelles, conservation de la nature, etc.) a amené les chercheurs à travailler sur de nouvelles problématiques comme l'impact environnemental du nautisme (Lloret et al., 2008; Francour et al., 1999; Milazzo et al., 2004; Peuziat, 2005, Le Corre, 2009), les conflits d'usages en mer et l'organisation des activités (Bossuet et Boutry, 2012; Dupilet, 2001 ; Le Guyader, 2012; Trouillet, 2006 ; De Cacqueray, 2011), ou encore la sécurité maritime (Lagane, 2008). Pour autant, sur un plan méthodologique comme thématique, la caractérisation et l'analyse des profils et des pratiques des plaisanciers restent un enjeu incontestable notamment au regard de l'importance du nautisme dans le développement local des territoires littoraux et de l'évolution des pratiques dans le domaine (Michot, 2008). 


\section{La nécessité d'une enquête départementale}

C'est dans ce contexte qu'une étude pour mieux connaître le profil et les pratiques des plaisanciers dans le Finistère a été entreprise en 2012 afin de compléter la connaissance des infrastructures et des capacités d'accueil ainsi que celle de la flotte.

Avec 1200 kilomètres d'un littoral très découpé par de nombreuses baies, criques, abers, le Finistère se situe parmi les départements français qui offrent la plus grande variété d'abris et de bassins de navi- gation (Lageiste, 1994). Bien adapté à la plaisance, le département n’a donc pas échappé au fort développement de l'activité à partir des années 1960 et de nombreux aménagements pour la plaisance y ont été réalisés depuis. Ainsi en 2012, le Finistère compte 373 sites de mouillage, 14 ports structurants (figure 1) et plus de 21000 plaisanciers (Le Berre, 2010). Les enjeux économiques y sont également considérables car ce secteur d'activité (tous secteurs nautiques confondus) comporte 505 entreprises, 2918 emplois Equivalents Temps Plein (ETP) et un

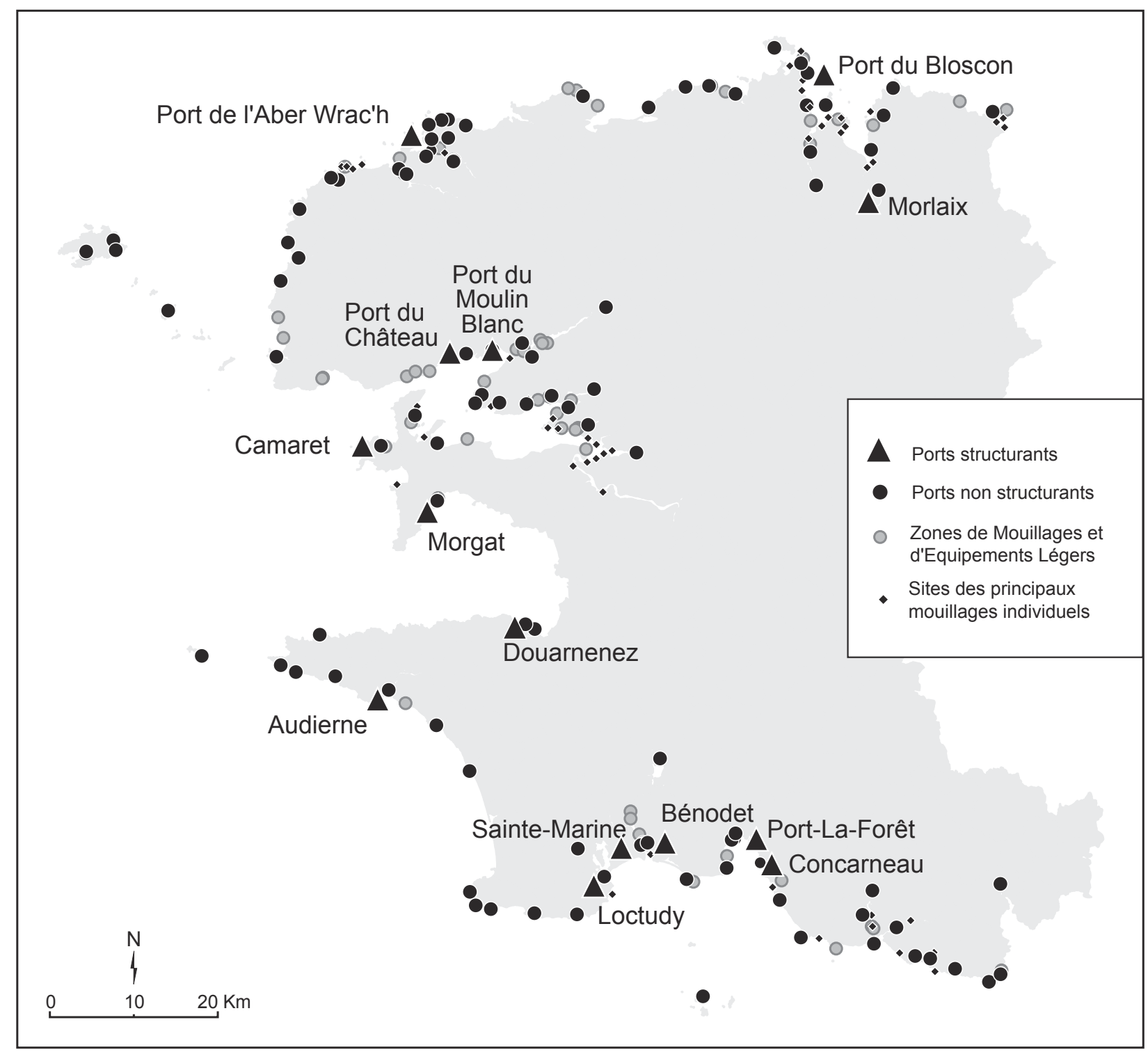

Figure 1 : Ports structurants et principaux sites de mouillages du Finistère Structuring marina and main mooring in Finistere 
chiffre d'affaire de 275 millions d'euros (Nautisme en Finistère, 2012).

\section{UNE ENQUÊTE EN LIGNE POUR UN ÉTAT DES LIEUX DÉPARTEMENTAL}

La principale difficulté pour disposer d'un échantillon représentatif de la diversité des pratiques à l'échelle d'un département, réside dans le nombre important et la variété des structures de plaisance (figure 1) ainsi que dans le caractère relativement individuel des pratiques. La structuration complexe de l'activité rend difficile la diffusion d'un questionnaire auprès des pratiquants. Ainsi, dans la littérature scientifique, différents moyens d'administration sont expérimentés pour mettre en ouvre ce type d'enquête auprès de plaisanciers : l'expédition par voie postale (Bernard 1993, Hee-Chan, 2003; Sidman, 2005; Sonnic, 2005), les enquêtes en faceà-face (Brigand et al. 2010; Gray et al. 2011 ; Peuziat 2005 ; Retière, 2003) ou par voie téléphonique (Odit France, 2008). Ces techniques ont été écartées à cause de leur coût important ou des difficultés liées à leur mise en œuvre à l'échelle du département. En France, les coordonnées des plaisanciers sont en effet très difficiles à obtenir auprès des différentes associations et gestionnaires contrairement aux États-Unis par exemple (Sidman et al., 2005).

Nous nous sommes donc orientés vers la diffusion en ligne d'un questionnaire, considéré comme un moyen d'enquête rapide et peu coûteux s'appuyant sur la diffusion des nouvelles technologies de l'information et de la communication (Frippiat et Marquis, 2010).

\section{Élaboration du questionnaire}

Lélaboration du questionnaire a été réalisée dans une démarche de co-construction avec les représentants de la plaisance et des usagers (9 associations locales de plaisanciers et 2 associations départementales) afin d'en faire valider le contenu et de garantir son appropriation par les usagers. Le questionnaire ainsi validé par le groupe de travail a été finalisé fin novembre 2012. Pour répondre aux préoccupations des acteurs, il aborde un vaste panel de sujets au travers de 56 questions, regroupées en 6 thématiques :

- Connaissance de la flotte (type d'embarcation, motorisation, âge, statut, armement...)
- Entretien du navire (budget et modalités d'entretien du bateau);

- Connaissance des plaisanciers (âge, Professions et Catégories Socioprofessionnelles, etc.);

- Pratiques et destinations (modalités et temporalités des pratiques nautiques, zones de navigation, fréquentation des îles);

- Connaissances générales sur la gestion et la réglementation (organisme de gestion portuaire et de gestion de l'environnement);

- Attentes et évolutions (perspectives de changement de port ou de pratiques);

Le logiciel d'enquête LimeSurvey a été utilisé ${ }^{2}$ pour concevoir le questionnaire en ligne et le diffuser. Ce logiciel libre (open-source) nous a permis de créer une interface simple, sobre pour l'utilisateur et garantissant l'anonymat des réponses. Après avoir testé le questionnaire auprès d'une vingtaine de plaisanciers afin de lever d'éventuelles incohérences ou des incompréhensions, le questionnaire a été mis en ligne le 10 décembre 2012 pour une durée de 6 mois.

\section{Une diffusion encadrée}

Afin d'enquêter le maximum de plaisanciers des différentes structures de plaisance finistériennes, un inventaire de tous les gestionnaires de ports et de mouillages, ainsi que des associations de plaisanciers a été établi. L’Union Nationale des Associations de Navigateurs (UNAN) et la Fédération Nationale des Pêcheurs Plaisanciers Sportifs de France (FNPPSF), structures fédérant plusieurs associations de plaisance, ont fourni une liste de leurs adhérents. Les contacts des associations n'adhérant pas à ces structures, ont été renseignés par Nautisme en Finistère (NEF) et complétés par des recherches auprès des communes. Au total, 123 associations ont ainsi été recensées et contactées dans le Finistère (figure 2).

Un courriel indiquant le lien d'accès au questionnaire en ligne a alors été expédié à tous les présidents d'associations et gestionnaires des structures de plaisance pour qu'ils le diffusent auprès de leurs adhérents ou clients. Les journaux locaux ont été sollicités (Ouest-France, Le Télégramme) ainsi que quelques sites en ligne spécialisés (Bretagne info

2. Site du logiciel d'enquêtes en ligne : [https://wwww.limesurvey.org/frr]]. 
Figure 2 : Co-construction et diffusion du questionnaire Join construction and dissemination of the survey

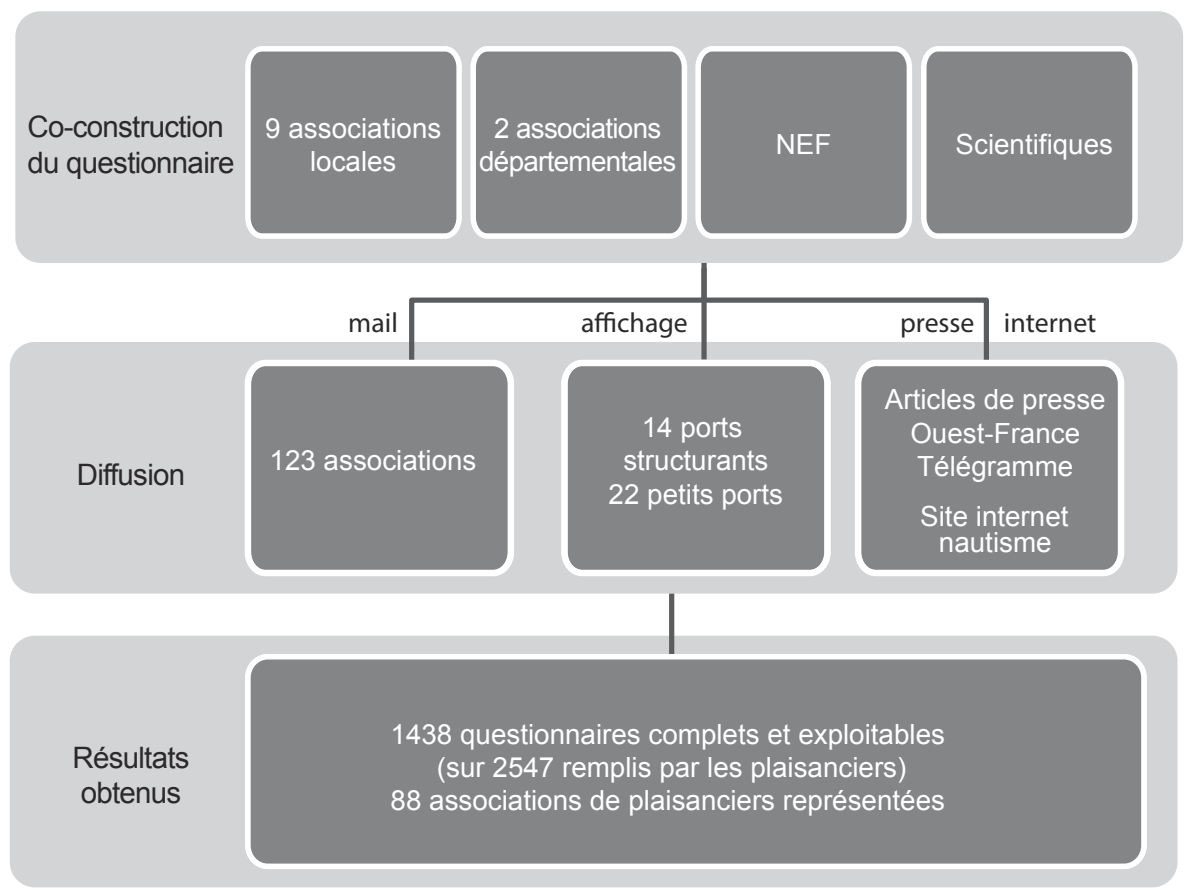

nautisme, NEF...) afin de diffuser l'information le plus largement possible.

Au total, 2547 questionnaires ont été renseignés. Parmi ceux-ci, 1109 n'ont pu être comptabilisés car ils n'étaient pas complets. Parmi les questionnaires complètement renseignés, très peu de réponses paraissaient incohérentes aussi, au final, 1438 questionnaires ont pu être exploités : 946 renseignés par des plaisanciers membres d'une des 88 associations qui ont bien voulu participer à l'étude et 492 renseignés par des plaisanciers n'appartenant à aucune association.

\section{Le redressement}

Les enquêtes auto-administrées, comme les enquêtes en ligne, fournissent des échantillons non représentatifs ou aléatoires car il n'est pas possible de contrôler l'échantillon qui va répondre. Des redressements statistiques sont possibles pour rétablir la représentativité d'un échantillon interrogé : il est alors nécessaire de disposer de variables en commun entre une population mère (proche ou égale au nombre de propriétaires de bateaux à flot) et l'échantillon traité (Escofier et Pagès, 2008).

\section{Exploitation des résultats de l'observatoire Bountîles NEF}

Quelques chiffres existent sur le nombre et le type de bateaux, cependant la plupart sont issus des données d'immatriculations de bateaux (DGITM). Or, ces données sont difficilement accessibles à l'échelle du département et tous les navires, y compris les navires inutilisables, y sont comptabilisés (Peuziat et Le Berre, 2015). D'autres sont issues des ports structurants (DGITM, 2010), mais, dans ce cas, les données des petits ports et mouillages ne sont pas renseignées. Dans le Finistère, les données les plus pertinentes sur ce sujet apparaissent donc être celles de Bountîles NEF, l'Observatoire de la fréquentation des mouillages de plaisance des côtes du Finistère (Brigand et al., 2005). Mis en place en 2004, il a pour but de "produire une estimation fiable et instantanée du nombre de mouillages et du type d'embarcations présentes sur l'ensemble du littoral finistérien et d'en avoir une représentation spatiale précise et incontestable ». Il repose sur des survols aériens réalisés à basse altitude tous les 5 ans. Ainsi le survol du 25 juillet 2009 a permis de comptabiliser, 21238 embarcations de plaisance (figure 3). Chaque type d'embarcation a été identifié et classé selon une typologie (voilier, embarcation à moteur, canot, pneumatique...), 


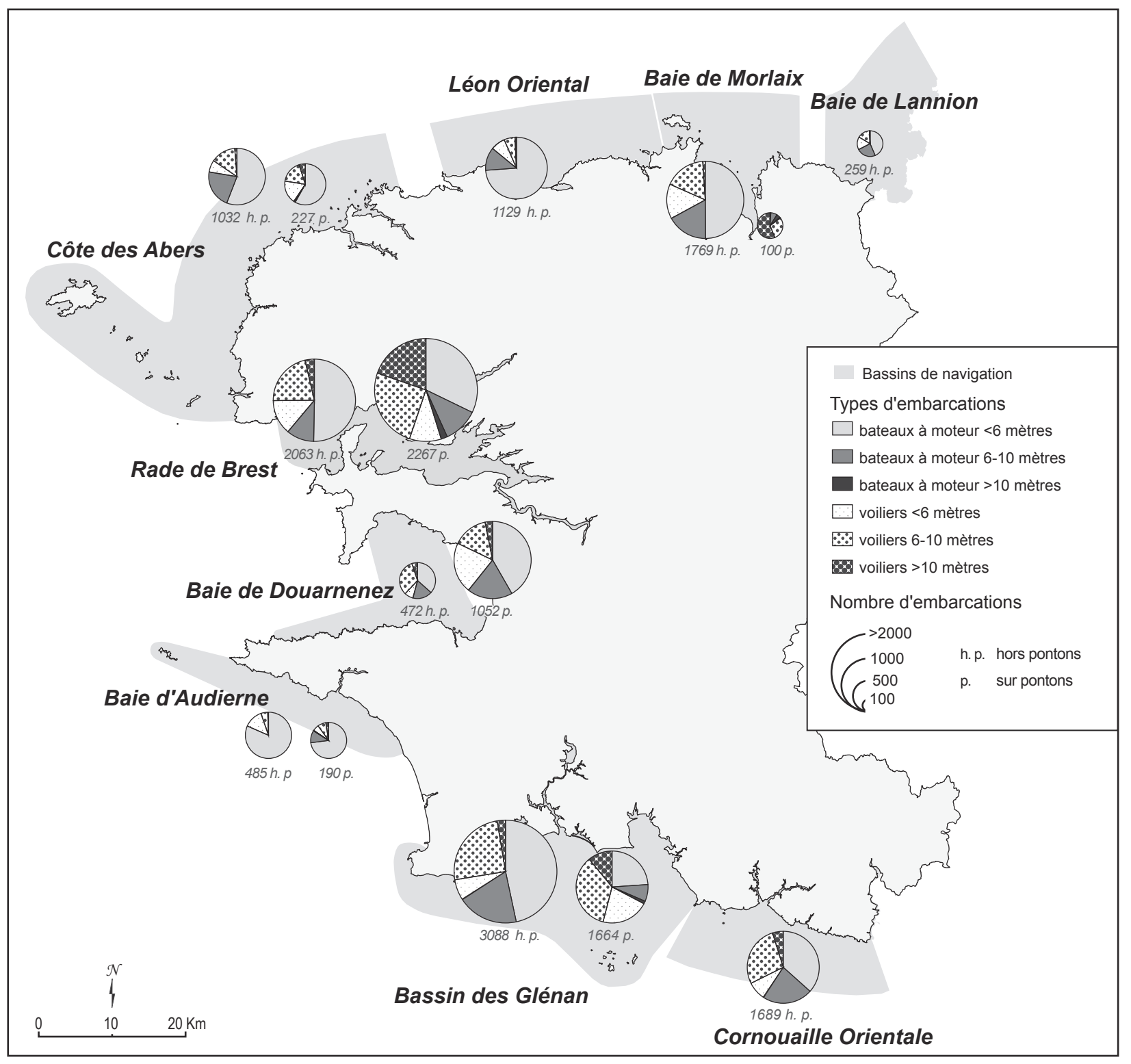

Figure 3 : Répartition du type d'embarcation par bassin de navigation (Bountîles, 2009) Distribution of type of boat by yachting area (Bountîles, 2009)

une taille et un type d'amarrage (ponton, corps-mort, quai, aucun). Les bouées sans bateau sont également comptabilisées.

L'échantillon issu de l'enquête en ligne et les données de l'observatoire Bountîles NEF ont en commun plusieurs critères (taille, type de propulsion, amarrage, bassin de navigation). Des pondérations ont été appliquées aux individus pour augmenter le poids de ceux appartenant à des groupes sous-représentés dans l'échantillon interrogé par rapport à la population-mère et, parallèlement, pour réduire le poids de ceux qui sont surreprésentés en utilisant un tirage aléatoire avec remise.

\section{Le traitement par bassin de navigation}

Les données de l'observatoire Bountîles NEF ont permis d'envisager un redressement à l'échelle du bassin de navigation (figure 3 et 4 ). Ce découpage géographique est une notion définie par le SEATL ${ }^{3}$

3. Service d'Étude et d'Aménagement Touristique du Littoral créé en 1966 au sein du ministère du tourisme et dissous en 1990. 
Figure 4 : Présentation de l'échantillon des plaisanciers enquêtés avant redressement.

Sample characteristics of respondents before adjustments

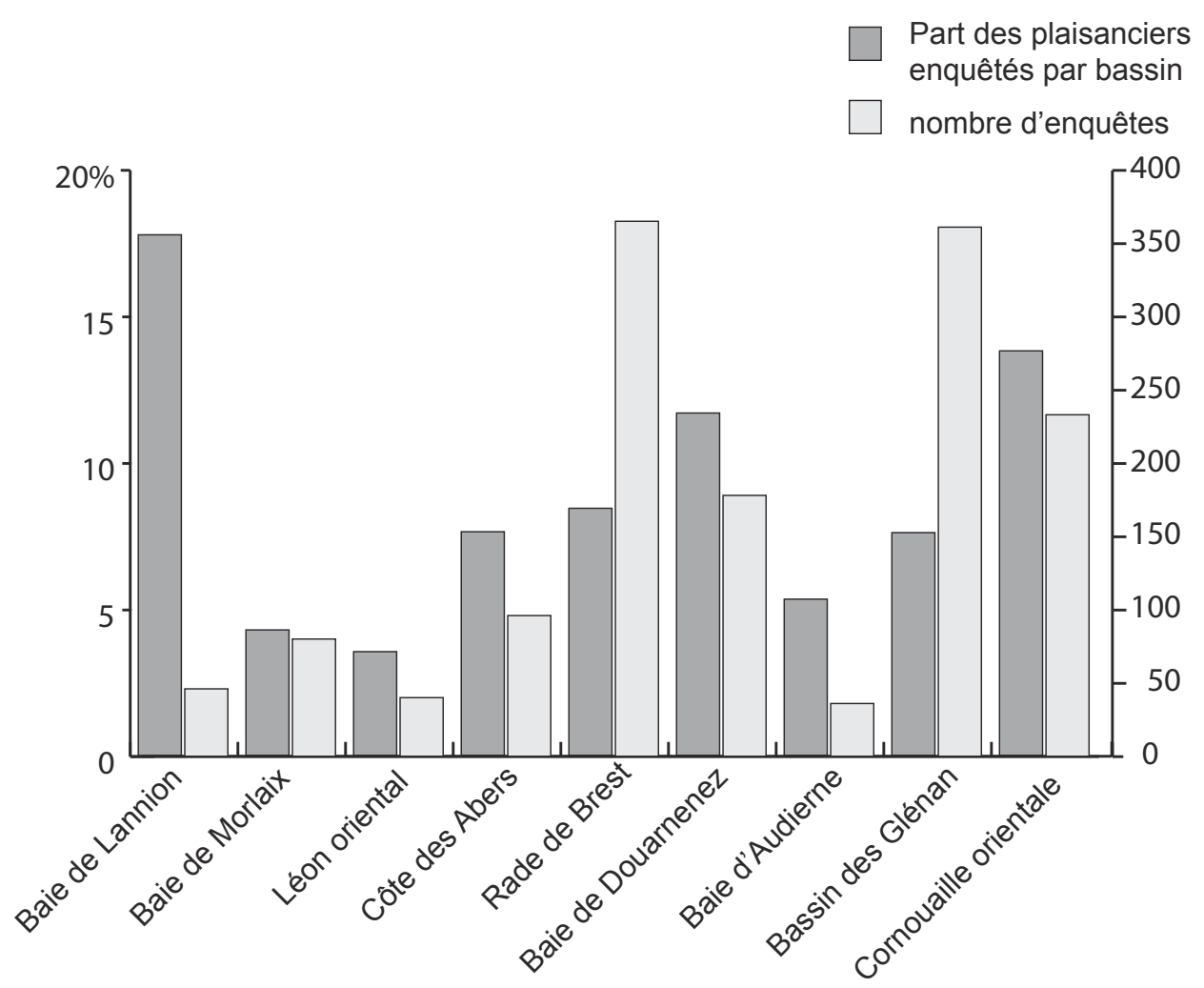

en 1982 en utilisant des critères géographiques tels que les pointes, les baies, les courants, les abris, délimitant ainsi un espace homogène doté des mêmes contraintes de navigation. Les découpages des bassins en Bretagne ont été modifiés de nombreuses fois depuis les années 1990 (Bernard, 1995; Lageiste, 1995; Sonnic, 2004; Tonini et Trouillet, 2005) mais, le plus utilisé actuellement dans les groupes de travail en Bretagne est celui issu des travaux de Baudelle et al. (2011). Nos résultats permettront notamment de vérifier si la réalité géographique matérialisée par ce découpage correspond à une réalité de pratique.

\section{Analyse statistique}

Dans un premier temps, le traitement a été réalisé par des tris à plat permettant de décrire globalement les variables, puis quelques croisements ont été effectués afin de montrer des relations entre deux variables. Ensuite, pour approfondir les traitements nous avons procédé à une Analyse des Correspondances Multiples (ACM). Cette méthode de description de données qualitatives est adaptée pour le traitement de données d'enquêtes (Escofier et Pagès, 2008). Elle permet de dégager des ressemblances entre individus du point de vue des variables et entre les modalités des variables. Une Classification Ascendante Hiérarchique (CAH) a ensuite été appliquée aux résultats de l'ACM. Elle autorise un regroupement des individus ayant un comportement similaire en classes. Ces analyses ont été réalisées à l'aide du logiciel libre $\mathrm{R}$ et de sa bibliothèque de fonctions FactoMineR. Cette méthode permet de différencier des groupes de plaisanciers aux caractéristiques relativement homogènes et d'en caractériser les profils et les pratiques.

\section{RÉSultats}

\section{Le plaisancier et son navire}

Les plaisanciers du Finistère ont une moyenne d'âge d'environ 60 ans, donc très supérieure à celle de la population du département qui est de 41 ans (INSEE, 2012). Ainsi $40 \%$ des plaisanciers ont entre 61 et 70 ans et $80 \%$ plus de 50 ans (figure 5). Sans surprise, la part des retraités est très impor- 


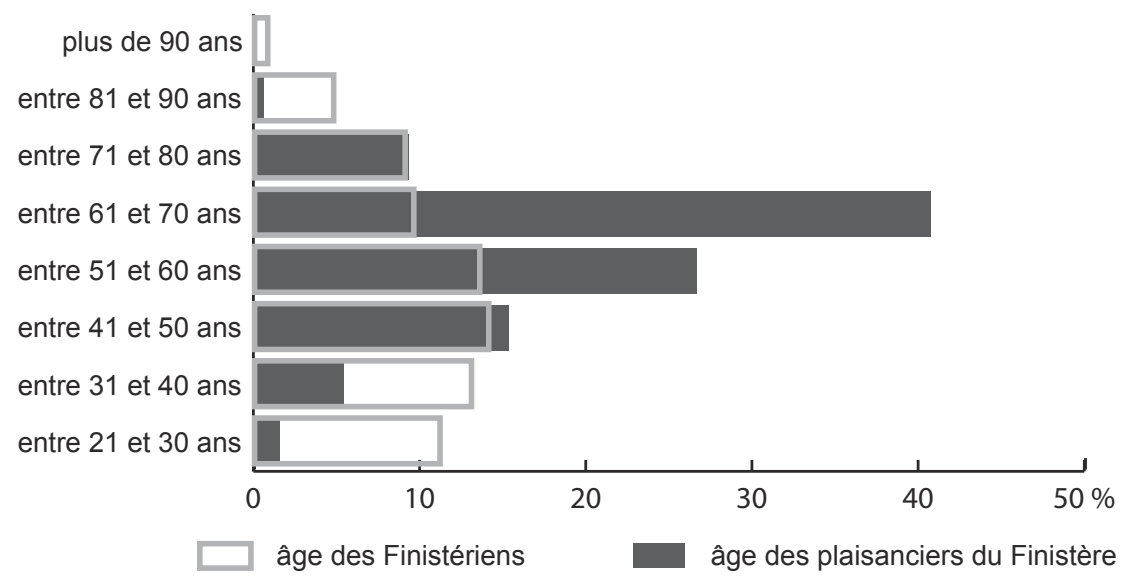

Figure 5: Distribution par tranche d'âge des plaisanciers et de la population du Finistère

Age distribution of Finistere's boater and population tante $(51 \%)$ tandis que $32 \%$ sont cadres, chefs d'entreprise ou exercent une profession intellectuelle supérieure. Il est également intéressant de constater qu'une grande majorité des plaisanciers est résidente $(72 \%)$ et que $60 \%$ d'entre eux font moins de 20 kilomètres pour accéder à leur bateau. $10 \%$ des plaisanciers sont franciliens et la quasitotalité des plaisanciers non résidants viennent de la moitié nord de la France.

$40 \%$ des bateaux déclarés sont à voile et $60 \%$ à moteur. La majorité des unités font moins de 7 mètres ( $55 \%$ ) et, parmi ceux-ci $45 \%$ des bateaux sont à moteur. Au-delà de 7 mètres, les bateaux sont majoritairement des voiliers (à $95 \%$ pour les plus de 10 mètres). La majorité des bateaux n'est pas amarrée dans les ports structurants (64\%).

Les résultats de notre enquête ont permis d'approfondir les données sur la structuration de la flotte. Lâge des voiliers est plus important que celui des bateaux à moteur (27 ans en moyenne contre 19) et les bateaux construits après 2000 sont essentiellement des vedettes (figure 6). Les plaisanciers changent relativement souvent de bateau (40\% ont changé 3 fois ou plus), en particulier les propriétaires de voiliers. $16 \%$ des plaisanciers enquêtés ont prévu de revendre leur bateau prochainement, soit pour acheter un bateau plus grand, soit parce qu'ils se jugent trop âgés, soit pour des raisons financières (loisir trop cher).

En effet, la moyenne des frais annuels du bateau s'élève à $2200 €(2900 €$ pour les bateaux amarrés dans les ports structurants et $1800 €$ pour les bateaux amarrés hors ports structurants). On peut toutefois préciser que $27 \%$ des plaisanciers disent dépenser moins de $1000 €$ par an. Quant à ceux qui dépensent plus de $6000 €$ par an, $98 \%$ sont propriétaires d'un voilier. Les frais engagés pour les bateaux à moteur sont probablement moindres car ils sont plus souvent stockés à domicile, contrairement aux voiliers qui restent à flot ou sur terre-plein.

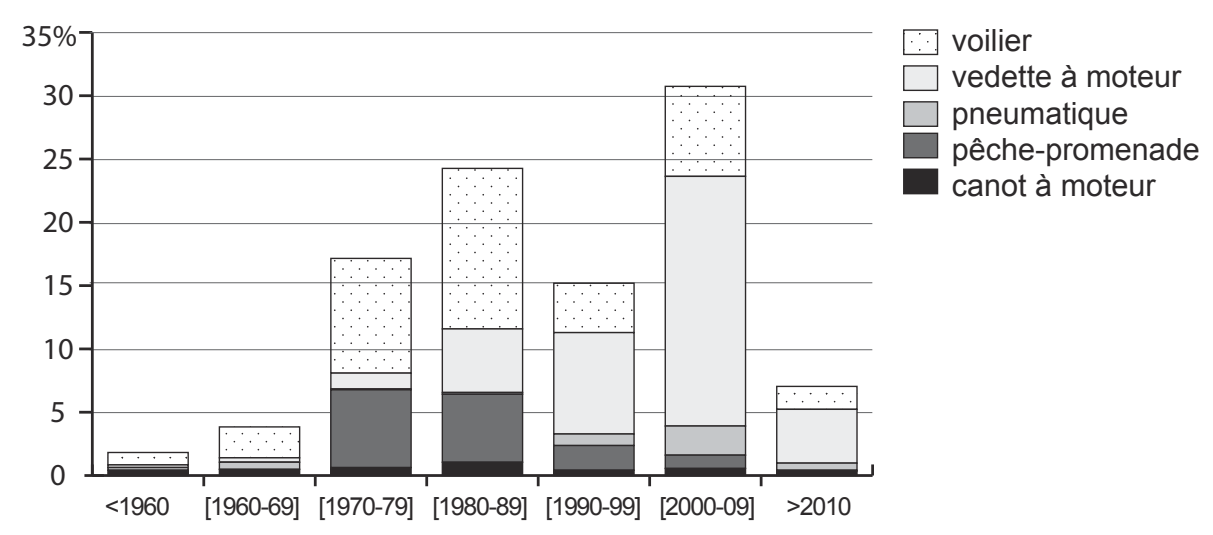

Figure 6 : Période de construction des navires par type tion by type 


\section{Les pratiques et zones de navigation}

La fréquence et la durée de sortie, de même que les activités pratiquées apparaissent très variées. Les propriétaires de bateaux à moteur sortent en majorité à la journée ou à la demi-journée (figure 7), seuls les propriétaires de voiliers sortent fréquemment sur de plus longues périodes. On peut constater que la grande majorité (54\%) des plaisanciers déclare sortir en mer entre 10 et 40 jours par an, $25 \%$ d'entre eux sortent entre 50 et 100 jours, seuls $10 \%$ sortent moins de 10 jours (figure 8). Les sorties sont plus fréquentes l'été : la totalité des plaisanciers sortent au moins une fois. Seuls $15 \%$ d'entre eux naviguent toute l'année. $40 \%$ des sorties s'effectuent en solitaire, $25 \%$ en couple et $20 \%$ avec des amis.

On constate également que les bateaux à moteur sortent très peu de leur bassin de navigation contrairement aux voiliers (exemple en rade de Brest figure 9). Les destinations principales des sorties en mer sont les zones de pêche à $45 \%$, les îles ou îlots à $20 \%$, tandis que $12 \%$ des plaisanciers annoncent naviguer sans destination précise. L'Archipel de Glénan est l'espace insulaire le plus fréquenté (36\% des plaisanciers) avec une part de fréquentation de bateaux à moteur supérieure à celle des autres îles (figure 10). Enfin, les zones de navigation hors du Finistère apparaissent relativement peu fréquentées à l'exception du Morbihan (17\% des plaisanciers).

\section{Les profils de plaisanciers}

\section{Analyse des Correspondances Multiples (ACM)}

Dix variables ont été retenues pour effectuer ce traitement : âge, professions et catégories socioprofessionnelles (PCS), bassin de navigation, type de bateau, habitabilité, taille, type de pratique, armement, utilisation du navire, adhésion à une associa-
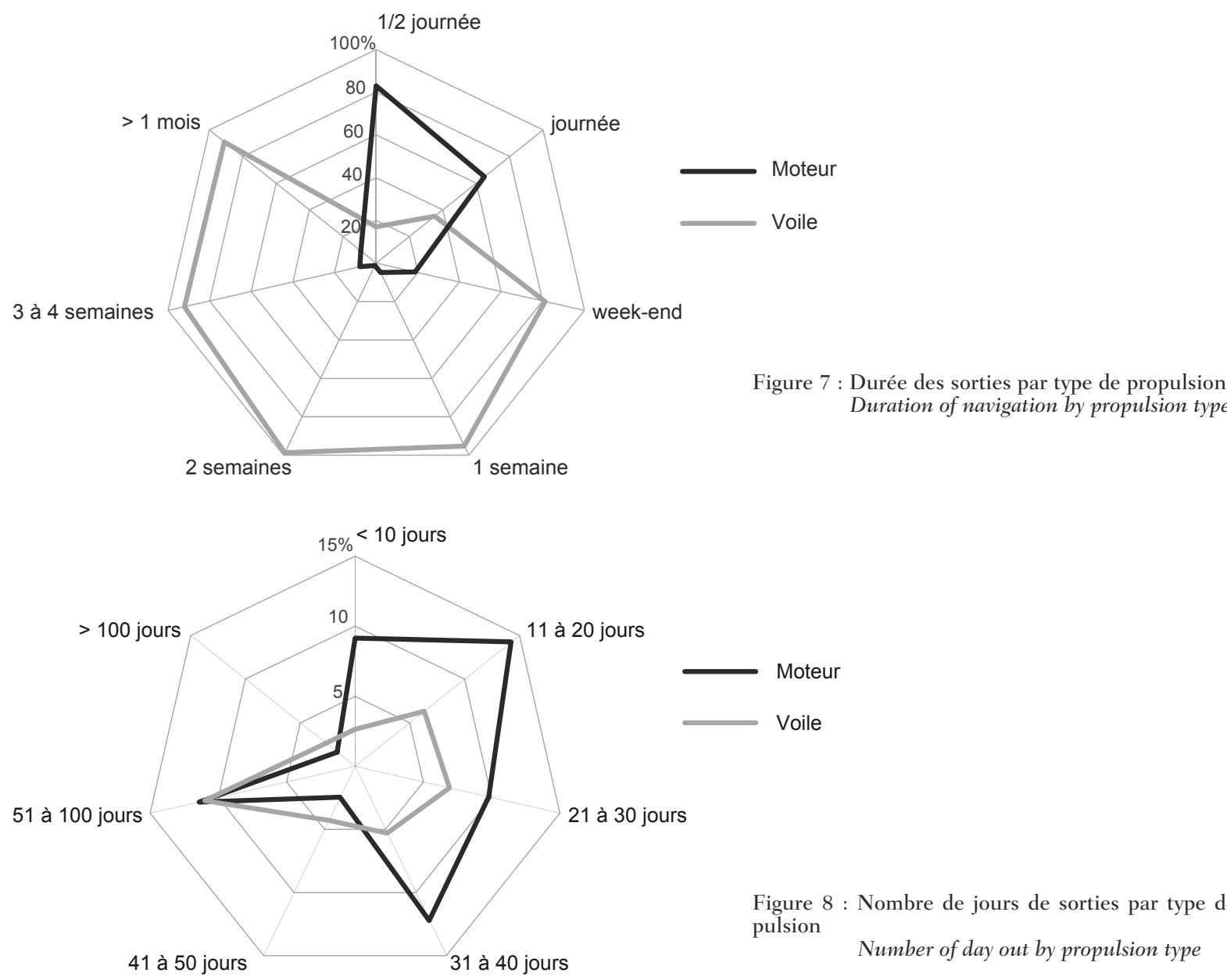

Figure 8 : Nombre de jours de sorties par type de propulsion

Number of day out by propulsion type 


\section{Voiliers}

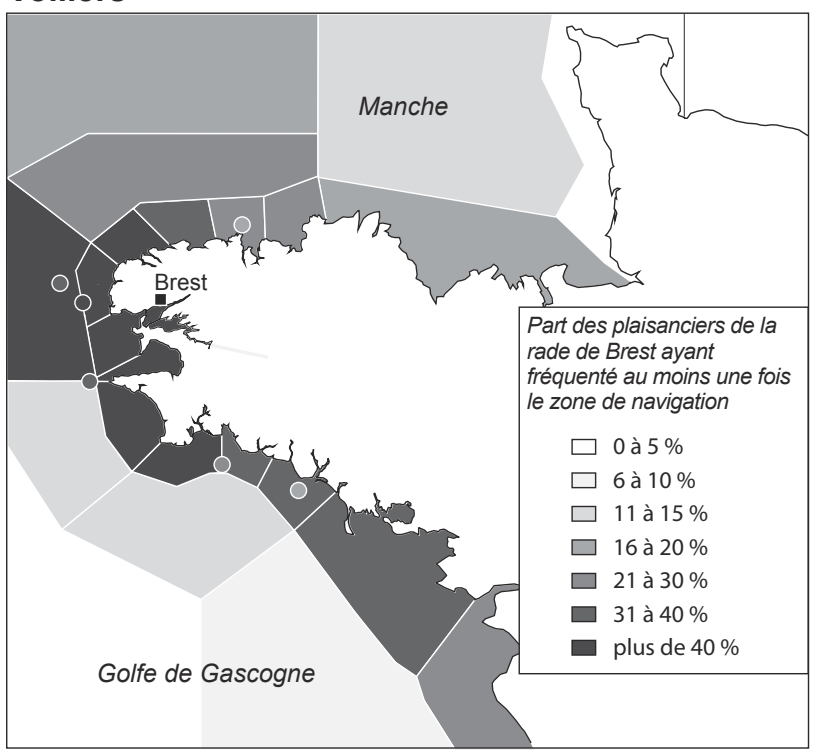

\section{Embarcations à moteur}

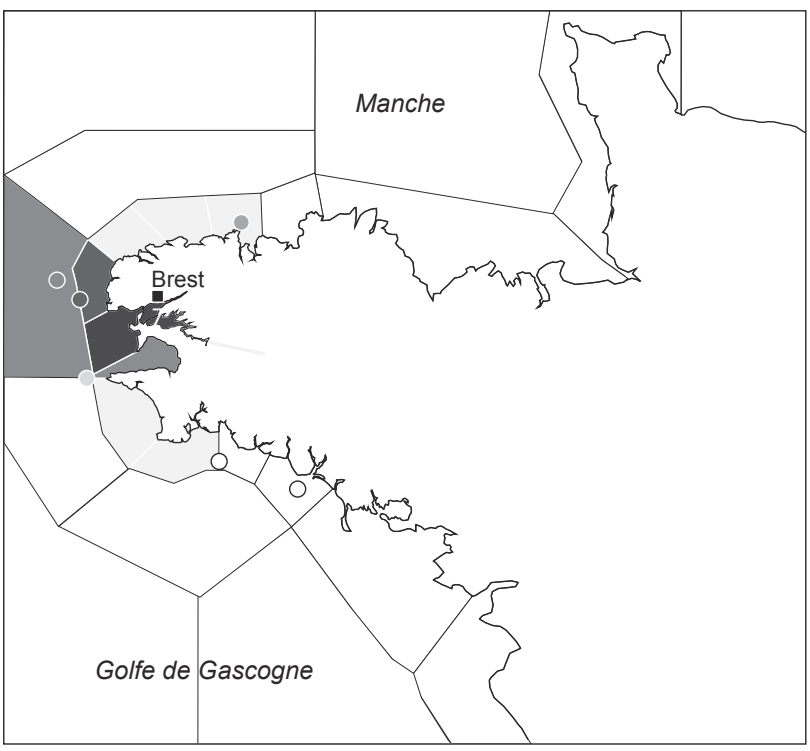

Figure 9 : Fréquentation des zones de navigation côtières par les plaisanciers de la rade de Brest selon le type de propulsion des embarcations

Use of coastal navigation areas by the boaters of the Bays of Brest according to leisure craft type

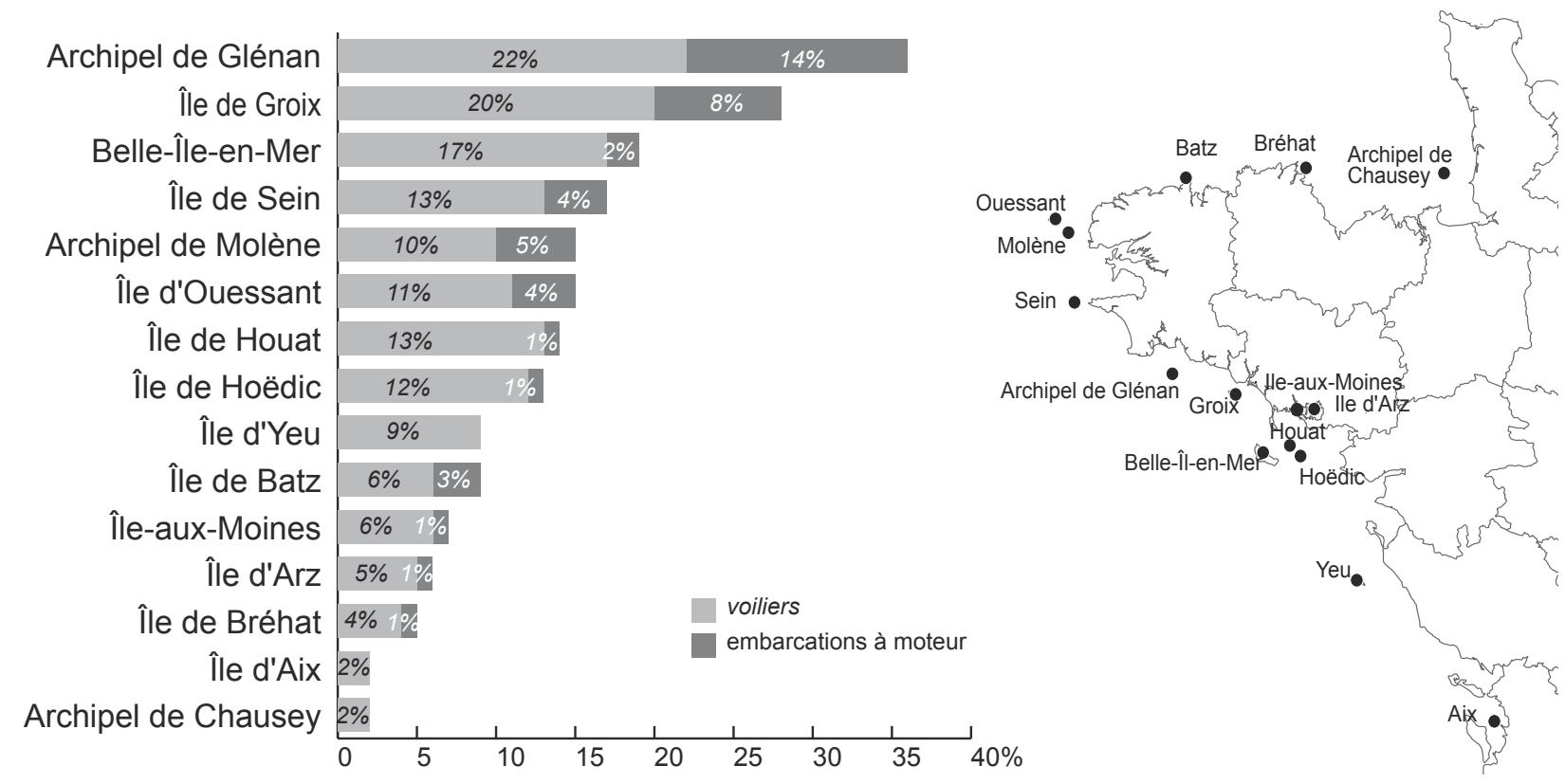

Figure 10 : Part des plaisanciers fréquentant les espaces insulaires par type de propulsion Part of boaters in island areas according to propulsion type

tion; les autres variables n'ayant que peu d'influence sur les catégories de plaisanciers.

Les deux premiers axes représentent 14,34\% (8,3 pour l'axe let 6,04 pour l'axe 2$)$ de l'inertie du nuage de points. Nous ne prenons pas en compte les autres axes trop peu représentatifs. Sur l'axe 1, les contributions sont basées essentiellement sur le navire, le type de bateau, le type de pratique, ainsi que la taille du bateau. Sur l'axe 2, les contributions sont basées essentiellement sur le profil du plaisancier : âge et PCS. 
En observant la figure 11, on voit que l'axe 1 discrimine les pratiques de bateau à moteur à droite, et à voile à gauche. À droite, on retrouve effectivement les pratiques plutôt motorisées (pêche, pêche sous-marine, pratique sportive motorisée et promenade en mer sur des petites unités). Celles proches de la voile sont regroupées à gauche (croisière, entraînement sportif et promenade en mer sur des unités plus grandes). L'axe 2 discrimine les profils des personnes avec, comme principale opposition, l'âge des pratiquants et leur catégorie professionnelle. Les modalités proches des plus jeunes sont regroupées en haut de l'axe 2 avec les pratiques sportives comme les sports motorisés (wakeboard, ski nautique) et la pêche sous-marine. À l'opposé, on retrouve en bas de l'axe des pratiques de pêche et de promenade en mer moins différenciées par l'âge des pratiquants.

\section{Classification Ascendante Hiérarchique (CAH)}

Afin de regrouper en classes les plaisanciers, une CAH a été utilisée. Cette technique se sert de résultats de l'ACM qui a préalablement repéré les variables et modalités structurantes afin de construire la CAH.

L'allure de l'arbre hiérarchique proposé par la $\mathrm{CAH}$, de même que le diagramme des inerties associées aux nœuds, suggèrent une partition en trois classes ou catégories (méthode de Ward). Cependant, selon notre expertise et l'interprétation de l'ACM, les résultats proposés ne mettent pas en évidence la catégorie généraliste (les occasionnels) pour lesquels les modalités sont partagées avec les autres catégories correspondant à quatre types de profils plaisanciers (ici replacés sur la représenta-

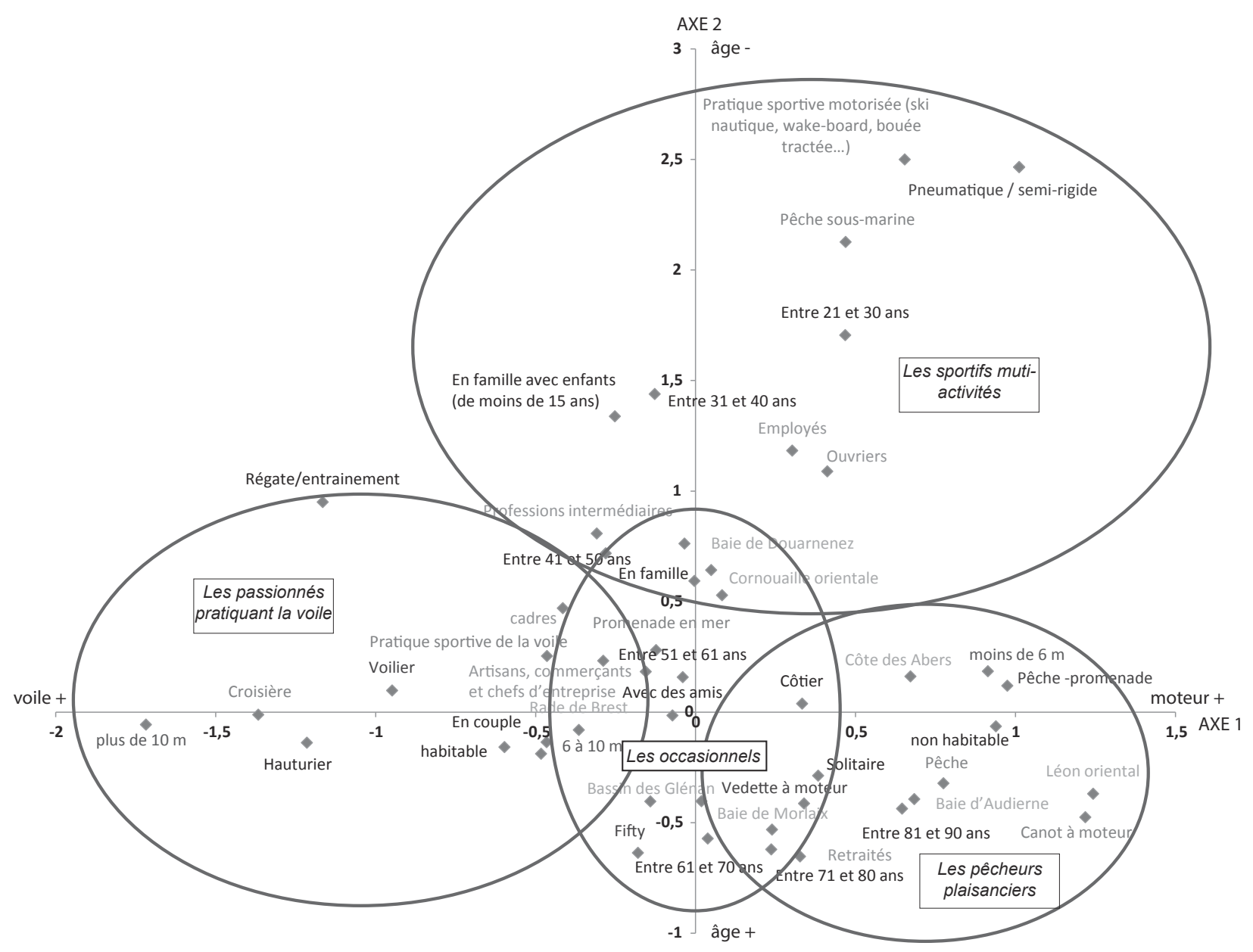

Figure 11 : Représentation plane du nuage des modalités issu de l'ACM Scatter plot visualization of Multiple correspondence analysis 
tion plane du nuage des modalités issu de l'ACM, figure 11). Le pourcentage de la population correspond à la part des catégories de plaisanciers proposées par la CAH. Les pourcentages des classes sont fournis à titre informatif mais ne représentent sans doute pas la part réelle des plaisanciers par type décrit.

- Les «passionnés pratiquant la voile » $(20,48 \%)$ affectionnent des bateaux adaptés à la croisière (78\% de la modalité), le plus souvent armés pour la navigation hauturière (67\% de la classe). Ce type de bateau est en très grande majorité à voile $(88 \%$ de la classe) et mesure plus de 7 mètres, pouvant atteindre des tailles de 15 mètres pour les plus grands $(97 \%$ des plus de 10 mètres sont dans cette classe). Ces bateaux sont présents essentiellement dans les ports de plaisance structurants ou dans des endroits très protégés comme des fonds de ria ou aber (Abers, Aven Belon...) avec un minimum de fond (leur tirant d'eau ne leur permettant pas d'échouer). La grande majorité de ces "voileux » ont plus de 50 ans $(76 \%)$ et viennent de catégories socioprofessionnelles élevées (cadres, artisans et chefs d'entreprises $56 \%$ et $30 \%$ de retraités).

Ces plaisanciers se distinguent des autres par leur rapport exclusif à leur loisir. Ils ont grandi avec en tête les exploits de Éric Tabarly ${ }^{4}$ ou les récits d'aventures de Bernard Moitessier ${ }^{5}$ (Roux M., 1994). Au regard du nombre de jours de sortie, une grande partie de leur temps libre est liée à la pratique ou à l'entretien du bateau. Il semblerait qu'ils aient été sensibilisés très tôt (souvent dès l'enfance ou l'adolescence) à la navigation dans les centres nautiques, les écoles de voile ou au sein du cercle familial. On peut noter que plus le bateau est gros, plus le nombre de sorties est faible mais, en contrepartie, plus la durée moyenne de sortie est élevée. Une minorité de ces passionnés pratiquent la régate.

- Les « occasionnels» $(36,48 \%)$ sont présents essentiellement dans les bassins de navigation les plus faciles d'accès en termes de navigation (rade de Brest, baie de Douarnenez, bassin des Glénan). Ils utilisent, le plus souvent, des vedettes à moteur ou des voiliers de taille «modeste » $(89 \%$ mesurent entre 6 et 10 mètres) équipés en navigation côtière. Leur principale activité est la promenade sans programme particulier. Ces balades sont le plus sou-

4. Avec comme date clé la victoire de la transat anglaise 1964.

5. Les ouvrages tels que «Vagabond des mers du Sud », « La longue route». vent réalisées en famille ou entre amis. La plupart d'entre eux ont entre 40 et 60 ans et viennent de catégories socioprofessionnelles assez variées. Cependant, le nombre de cadres est légèrement supérieur à la moyenne du Finistère et le nombre de retraités légèrement inférieur. Les résultats des enquêtes montrent que ces pratiquants sont surtout Finistériens. Les quelques résidants hors du département y possèdent une résidence secondaire. Leurs sorties dépendent généralement de la météorologie, la pluie étant un des principaux facteurs limitant.

- Les «sportifs multiactivité » $(4,82 \%)$ sont présents sur tout le territoire mais affectionnent, comme les « occasionnels », les bassins les plus simples en termes de navigation. Ils utilisent le plus souvent des pneumatiques ou autres bateaux à moteur de taille modeste, à bord desquels ils peuvent pratiquer plusieurs activités comme la chasse sous-marine, la pêche ou de temps en temps le ski nautique par exemple (100\% de la modalité). Leur pratique est donc souvent variée. Les moins de 40 ans sont les plus nombreux à pratiquer plus de deux activités à partir de leur bateau. Il serait intéressant de savoir si ces plaisanciers pratiquent également d'autres activités nautiques en dehors de la plaisance (planche à voile, surf, kite-surf). Ils naviguent entre amis et parfois en famille pour une balade en mer. Ils sont plus jeunes que la moyenne des plaisanciers. Leurs professions et catégories socioprofessionnelles sont assez variées avec une légère prédominance pour les cadres.

- Les «pêcheurs plaisanciers » $(38,23 \%)$ sont très présents sur tous les bassins de navigation du Finistère et sont majoritaires sur les bassins les plus hostiles en termes de navigation (côte nord, baie d'Audierne...). Ils utilisent le plus souvent des embarcations à moteur de petites tailles, inférieures à 6 mètres ( $86 \%$ de la classe) et non habitables, de type «pêche promenade » (96\% de la modalité), ou des vedettes à moteur. Leur moyenne d'âge est assez élevée et les "pêcheurs-plaisanciers » de plus de 70 ans ne sont pas rares. En grande majorité ils sont retraités $(70 \%)$, cependant les enquêtes ne nous permettent pas de connaître leur ancienne catégorie socioprofessionnelle. Ils sortent le plus souvent seuls $(60 \%$ de la classe) et de temps en temps en famille ou avec des amis. La plupart sont adhérents à une association de plaisanciers. 
Les sorties sont plutôt courtes, parfois moins d'une heure et ne dépassent jamais la journée. Il semble que, pour une partie d'entre eux, la pêche de plaisance soit une pratique de loisir exclusive à laquelle ils consacrent beaucoup de leur temps libre, ce qui en fait des plaisanciers qui sortent très régulièrement.

Ce sont majoritairement des «locaux » qui habitent à proximité du lieu d'amarrage de leur embarcation. Cela leur permet de sortir rapidement et facilement en fonction de la marée (contrainte importante en Finistère nord) et de la météo.

\section{Quelle évolution depuis 1992 ?}

À partir de l'enquête réalisée en 1992 par Nicolas Bernard (1993) dans le cadre sa thèse sur les ports structurants du Finistère, une comparaison peut s'envisager. Elle montre que la part des plaisanciers âgés de plus de 50 ans a augmenté de plus de 20 points entre 1992 et 2013 dans les ports structurants, passant de $58 \%$ à $80 \%$ (figure 12). Sans en avoir de réelle preuve, il est possible que l'on retrouve sensiblement la même population de plaisanciers mais plus âgée de 20 ans en 2013 qu'en 1992, avec sans doute peu de nouveaux arrivants.

L'augmentation du nombre de retraités entre 1992 et 2013 vient confirmer le vieillissement des plaisanciers. La part de retraités a augmenté de $20 \%$ aux dépens des autres PCS, à l'exception de celle des cadres et professions intellectuelles supérieures qui a très peu reculé.

En revanche les caractéristiques de taille des bateaux n'ont pas fondamentalement changé : les bateaux de moins de 6 mètres ainsi que ceux de 6 à 10 mètres sont présents à plus de $40 \%$ dans chaque port. Une augmentation de 7 points est observable sur les bateaux supérieurs à 10 mètres aux dépens des catégories 6-10 mètres.

Les activités les plus pratiquées restent les mêmes et dans les mêmes proportions (40\% pêche, $55 \%$ promenade, $5 \%$ voile sportive). En revanche, les plaisanciers les plus jeunes affectionnent davantage la multiactivité : $55 \%$ des jeunes de moins de 40 ans pratiquent trois activités différentes contre $15 \%$ pour les plus de 60 ans. Les plaisanciers sortant moins de 20 jours ont augmenté de 20 points (entre 1992 et 2013). Les profils "pêcheurs plaisanciers » et «passionnés » pratiquant leur loisir, de façon le plus souvent exclusive, semblent diminuer au profit des profils « occasionnels » ou « multiactivité ».

\section{Discussion}

\section{Limite du questionnaire}

La réalisation d'une enquête en ligne apparaît $a$ priori comme une solution simple, rapide et bon marché. Elle implique cependant un important travail de co-construction et d'animation. L'implication des réseaux d'associations de plaisance (UNAM, FNPPFS) a fortement contribué à la réussite du questionnaire. Une contrainte à ce type de démarche est alors de définir un contenu de questionnaire qui satisfasse tous les partenaires. De fait, les sujets traités ont été variés mais relativement peu approfondis. Des problématiques spécifiques à forts enjeux pour l'avenir de la plaisance devront encore être développées telles que la transmission intergénérationnelle des pratiques nautiques, la spatialisation des activités ou leur contribution à la gestion durable de l'environnement.

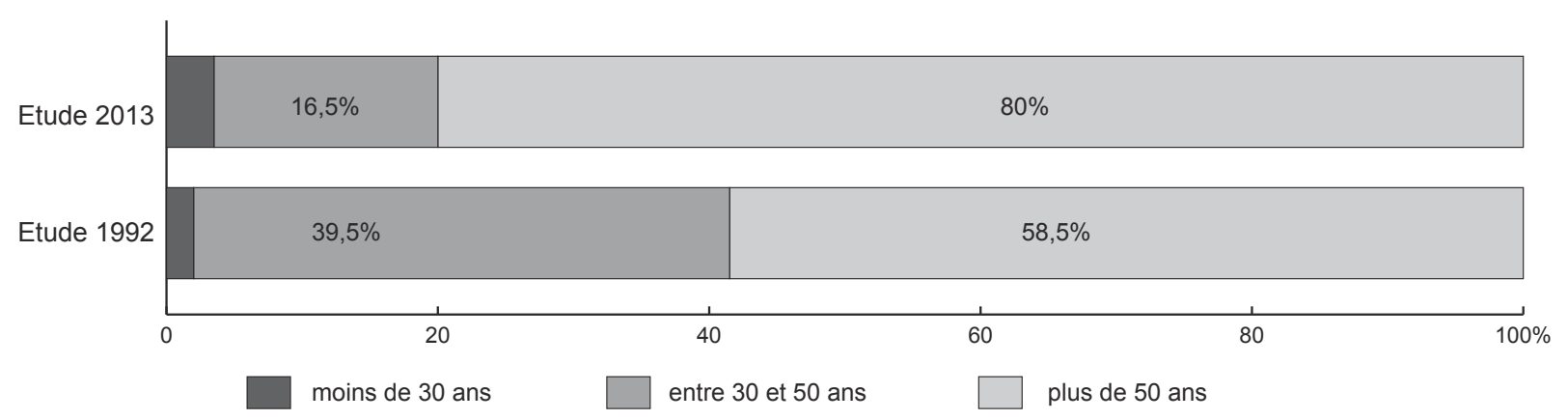

Figure 12 : Comparaison de l'âge des plaisanciers des ports structurants entre 1992 et 2013 (d'après Bernard, 1993). Age comparison of marina boaters between 1992 and 2013. 
Le taux d'équipement des ménages disposant d'internet à son domicile en France est beaucoup plus faible pour les seniors et les retraités (44,3\% et $30,7 \%$; source INSEE 2010) que pour l'ensemble de la population, ce qui pose un problème quand on s'adresse à une population relativement âgée telle que celle des plaisanciers finistériens. Cependant, dans le cadre de l'enquête réalisée, ces chiffres sont sans doute atténués par la forte proportion de retraités plaisanciers venant de PCS élevées ayant utilisé internet au cours de leur vie professionnelle.

Enfin la crainte d'une sur-sollicitation des plaisanciers par les enquêtes ou les inquiétudes quant à l'usage qui sera fait des résultats (régulation de certaines activités, restriction de liberté...), ont conduit certaines associations ou des gestionnaires de port à ne pas relayer le questionnaire auprès de leurs adhérents ou usagers, tandis que d'autres plaisanciers n'ont pas souhaité y répondre.

Le bilan de l'enquête en ligne reste néanmoins positif. Six pourcents des plaisanciers finistériens ont pu être enquêtés avec une bonne représentativité au regard de la population mère, même avant redressement de l'échantillon. Dans l'ensemble les associations et gestionnaires se sont impliqués et les plaisanciers ont manifesté des attentes fortes sur cette thématique puisque deux tiers d'entre eux ont laissé leurs coordonnées pour un retour de l'étude.

\section{Vers un changement de pratiques?}

\section{Un vieillissement incontestable}

Avant de parler d'un changement de pratiques, il semble intéressant de s'attarder sur l'évolution de la structure du profil des plaisanciers. Le résultat le plus marquant est sans conteste leur vieillissement. Effectivement, entre 1992 et 2013, la part des plaisanciers du plus de 50 ans a augmenté de 20 points (soit $80 \%$ des plaisanciers en 2013) dans les ports structurants. L'une des cause de revente de bateau les plus citées (qui concerne $20 \%$ de plaisanciers) est un âge jugé trop avancé pour poursuivre l'activité plaisancière sur le support actuel.

De fait, sans que des statistiques précises aient encore été établies sur ce point, d'après de nombreux responsables de ports de plaisance, les listes d'attente, bien qu'encore longues, semblent aujourd'hui se contracter rapidement. Ainsi, nombre de plaisanciers sollicités par les gestionnaires lors d'une libération de place refusent finalement cette attribution pour diverses raisons. On peut se demander s'il s'agit d'un phénomène conjoncturel, lié à la crise économique, ou bien d'une tendance de fond.

\section{Vers un nouveau modèle de plaisance?}

Ces tendances ne sont pas nouvelles : en 1993 $\mathrm{N}$. Bernard écrivait déjà que : « la tendance au nonrenouvellement des propriétaires de voiliers de croisière et le recul de leur moyenne d'âge sont les prémices d'un désengagement qui pourrait s'accentuer à terme : les plus jeunes sont de moins en moins attirés par l'acquisition d'un bateau, opération financière lourde qui interdit souvent la dispersion vers d'autres activités de loisirs (vacances à la montagne, voyages...)».

Vingt ans après, cette tendance semble s'être confirmée et généralisée sur la plupart des supports. Le contexte économique ne facilite effectivement pas l'accès à la propriété d'un bateau pour les plus jeunes mais d'autres facteurs doivent être considérés comme l'évolution des structures familiales (divorces, décohabitation...) et la diversification de l'offre de loisirs. Ainsi, de 1990 à 2005, le nombre d'associations sportives en France est passé de 150000 à 170000 , le nombre de pratiquants en club de 12 millions à 14 millions, et les pratiques libres sont estimées en 2005 à 20 millions de pratiquants (Ferréol, 2008). Ces évolutions sociales laissent donc probablement moins de place à la plaisance, loisir exigeant, qui demande un investissement en temps et un engagement financier important. Malgré des événements aussi médiatisés que les grandes courses au large (Vendée Globe, route du Rhum...), l'engouement pour cette pratique semble donc reculer.

Ce constat pose de réels questionnements aux acteurs de la filière nautique ainsi qu'aux collectivités territoriales et gestionnaires des équipements de plaisance : quel avenir du nautisme comme vecteur de développement économique des zones côtières? Quelle légitimité pour certains projets de création ou d'extension de port de plaisance? Quels investissements allouer à l'entretien des ports de plaisance (pontons, piliers, dragages etc.) dans un contexte de restriction des finances publiques? Pour reprendre l'exemple du Finistère, l'élaboration du $5^{\mathrm{e}}$ Livre Bleu 
(document stratégique du nautisme finistérien) avec les acteurs du nautisme est l'occasion d'affirmer « l'exigence de renouvellement, voire de ruptures, dans les modes d'organisation et de fonctionnement, afin de les adapter aux évolutions » de ce nouvel environnement (Livre Bleu 2015-2020).

\section{Conclusion}

L'étude des caractéristiques et des usages des plaisanciers du Finistère permet de mieux comprendre l'évolution des pratiques à un moment charnière où la génération qui a connu « le grand essor plaisance » raccroche progressivement cirés et bottes, et où une nouvelle génération de plaisanciers, porteuse de nouveaux comportements, reste finalement assez discrète dans les ports et les zones de mouillage.

Ainsi, le fort vieillissement observé matérialise le faible renouvellement des pratiquants de la plaisance et soulève d'importantes questions concernant les besoins d'infrastructures et de services. À ce propos, il est important de rappeler que les listes d'attente dans les ports de plaisance sont une exception française. Dans la majorité des ports d'Europe du nord, souvent privés, les taux d'occupation sont inférieurs à $90 \%$ et les listes d'attentes se sont énormément rétractées ces dernières années (Earth to Ocean, 2011).

De plus, l'étude montre que les plus jeunes ont une approche différente de leurs aînés et sont attirés par la multiactivité. En effet, davantage qu’à un manque de place dans les ports, le succès des bateaux transportables apparaît lié à de nouvelles pratiques répondant à des critères de mobilité (Perras, 2012). Elle se reflète aussi dans l'explosion de pratiques individuelles de sports nautiques de glisse (kite surf, surf, planche à voile, stand-up paddle...) qui répondent sans doute à une nouvelle forme d'évasion moins lourde financièrement et logistiquement, procurant de nouvelles sensations, véhiculant une image de sport «fun » et une autre forme de liberté. En parallèle l'offre de location de voilier dans des bassins de navigation dépaysants et au climat attractif (Croatie, Grèce, Antilles...) se multiplie (Desse, 2000).

Conscients de ces changements, les actuels gestionnaires sont contraints de réfléchir à l'évolution des infrastructures portuaires ainsi qu'aux services à développer pour attirer une nouvelle génération de plaisanciers (location, co-navigation...). Ces interrogations montrent que l'observation et le suivi des attentes des plaisanciers est un sujet qui mérite une attention soutenue afin d'anticiper de probables changements susceptibles d'avoir des répercussions sur une activité dont l'importance, tant économique que sociale et culturelle, n'est plus à démontrer, notamment pour les territoires à économie résidentielle comme le Finistère.

\section{Remerciements}

Les auteurs tiennent à associer les étudiants de Master 2 «Expertise et gestion de l'Environnement Littoral » (2012-2013), Nolwenn Cozannet, Vincent Le Viol, Thomas Pavy, Elouan Meyniel et Olivier Morio, du master Sciences de la mer et du littoral de l'IUEM-UBO ayant participé à l'étude sur laquelle s'appuie cet article. Nous associons également à ce travail, en les remerciant particulièrement, M. François Arbellot et Olivier Poncelet, de Nautisme en Finistère pour avoir initié et soutenu cette recherche. Nous tenons également à remercier vivement les deux relecteurs anonymes dont les remarques très constructives ont contribué à améliorer sensiblement cet article.

\section{Bibliographie}

Baudelle G., Sonnic E., Alkan D., Quantin P.-Y., Duhayon J.-J., 2011 . L'accueil des navires de plaisance dans la perspective d'une GIZC. Rapport UMR ESO CNRS 6590 - Université de Rennes 2, CETE de l'Ouest, 248 p.

BERNARD N, 2000. Les ports de plaisance-Équipements structurants de l'espace littoral, Paris, L'Harmattan, $126 \mathrm{p}$.

Bernard N., 1993. Ports de plaisance et structuration de l'espace littoral finistérien, Thèse de doctorat de Géographie, Université de Bretagne Occidentale, 275 p.

Bernard N., 1995. Port de plaisance : pour quelle utilisation de l'espace maritime?, Norois, ${ }^{\circ} 165$, p. 225-234.

Bernard N. (dir.), 2005. Le Nautisme, acteurs, pratiques et territoires, Rennes, PUR, coll. «Espace et territoires », 332 p.

Bossuet L., Boutry O., 2012. Conflits d'usage et de voisinage autour de la ressource en eau, Illustration à partir du littoral charentais, Economie rurale, $\mathrm{n}^{\circ} 332$, p. 74-87.

Brigand L., Le Berre S. et al., 2005. État des lieux des mouillages organisés et forains utilisés par les plaisanciers le long des côtes finistériennes, rapport Laboratoire LETG-Brest 
Géomer, université de Bretagne Occidentale/Nautisme en Finistère, 199 p.

Brulé-Josso S., 2012. Les plaisanciers et le vrai marin, Ethnologie française, vol. 42, $\mathrm{n}^{\circ}$ 4, p. 733-745.

Clerc Giraudo C., 2002. Les ports de plaisance sur le littoral de Provence-Côte d'Azur. Étude géographique, L'information géographique, vol. 66, $\mathrm{n}^{\circ} 1, \mathrm{p} .81-88$.

Conseil Général du Finistère, 2015. Livret Bleu du nautisme, Principes, enjeux et plan d'action, Nautisme en Finistère, Session plénière 29-30 janvier 2015, Pleyben, 62 p.

Créac'h C., 2003. Les usages sociaux des voiliers. Enjeux culturels des modes d'appropriation de la navigation, Thèse de doctorat en sociologie, université de Nantes, 534 p.

D’Aboville G., 2013. De l'urgence d'une grande enquête sociologique sur la plaisance, Le Marin, Dossier spécial, Port de plaisance, mars 2013, p. 7.

De Cacqueray M., 2011. La planification des espaces maritimes en France métropolitaine: un enjeu majeur pour la mise en cuvre de la Gestion Intégrée de la Mer et du Littoral, Thèse de doctorat de géographie, université de Bretagne Occidentale, LETG-Brest Géomer UMR6554, 554 p.

Desse M. 2000. L'impact économique de la plaisance à la Martinique (France), Annales de Géographie, vol. 109 n 613, p. 306-316.

DGTIM, 2013. La plaisance en quelques chiffres, 21 p. [http:// www.developpement-durable.gouv.fr/IMG/pdf/plaisqqc2013. $p d f]$.

Dupilet D., 2001. Le règlement des conflits d'usage dans la zone côtière entre pêche professionnelle et autres activités, rapport ministériel, 57 p. [http://agriculture.gouv.fr/IMG/pdf/rapport_dupilet-0.pdf].

Earth to Ocean Ltd. Consultant, 2011 , Strategic Guidance for the Solent: water based recreation, Solent Forum, $21 \mathrm{p}$.

Escofier B., PAGÈs J.-L., 2008, Analyses factorielles simples et multiples, objectifs méthodes et interprétation, Dunod, 284 p.

Falt P., 1981. Les usages sociaux de la croisière, in Pociello P. (dir.), Sport et Société. Approche socio-culturelle des pratiques, Paris, Vigot, p. 261-284.

Fédération des Industries Nautiques, Fondation BénéTEAU, 2014, Les attentes des futurs plaisanciers, rapport FIN, $27 \mathrm{p}$.

Ferréol G., 2008. Loisirs, sports et sociétés : regards croisés, Besançon, Presses universitaires de Franche-Comté, 166 p.

Francour P., Ganteaume A., Poulain M., 1999. Effects of boat anchoring in Posidonia oceanica seagrass beds in the Port-Cros National Park (north-western Mediterranean Sea), Aquatic conservation : marine and freshwater ecosystems, $n^{\circ}$ 9, p. 391-400.

Frippiat D., Marquis N., 2010. Les enquêtes par Internet en sciences sociales : un état des lieux, Populations, vol. 2, $n^{\circ} 65$, p. 309-338.

Gray, D.L., Canessa, R., Keller C.P., Dearden P., Rollins R., 2011. Spatial Characterization of Marine Recreational Boating: Exploring the Use of an on-the-Water Questionnaire for a Case Study in the Pacific Northwest, Marine Policy, vol. 35, n³, p. 286-298.
Hee-Chan L., 2003. Estimating Recreational Boater Expenditures on Trips and Boating Use in a Wave Survey, Leisure Sciences, vol. 25, n 4, p. 381-397.

INSEE, 2012. Évolution et structuration de la population du Finistère, 19 p. [http://www.statistiques-locales.insee.fr/ FICHES/DL/DEP/DL_DEP29.pdf].

Lagane Y., 2008. Peut-on améliorer la sécurité de la plaisance et des loisirs, La revue Maritime, $n^{\circ} 482$, p. 22-25

LAGEISTE J., 1994. Les ports de plaisance en Bretagne : protagonistes $d u$ développement touristique?, Thèse de doctorat de géographie, Université Paris-Sorbonne Paris 4. 235 p.

Lageiste J., 1995. Le nautisme en Bretagne, Norois, n 166 , p. $443-452$.

Lageiste J., 2000. Ports de plaisance et navigation de plaisance sur le littoral du Nord-Pas-de-Calais, Hommes et Terres $d u$ Nord, $n^{\circ} 1$ p. 27-35.

Le Berre S., 2010. Bountîles Nautisme en Finistère, Observatoire de la fréquentation des mouillages de plaisance des côtes du Finistère, Résultats de la campagne aérienne du 25 juillet 2009, Rapport laboratoire Géomer LETG/UMR 6554 CNRS/université de Bretagne Occidentale, Nautisme en Finistère, 13 p.

Le Berre S., Courtel J., Brigand L., 2010. Étude de la fréquentation nautique du Bassin d'Arcachon, Laboratoire LETG-Brest Géomer UMR 6554, Direction régionale des affaires maritimes, Aquitaine, 94 p.

Le Bras C., Lacombe P., 2011. Les navigatrices de course au large. Une socialisation professionnelle spécifique, Ethnologie française, vol. 41, n 4, p. 717-726.

Le Corre N., 2009. Le dérangement de l'avifaune sur les sites naturels protégés de Bretagne : état des lieux, enjeux et réflexions autour d'un outil d'étude des interactions homes/ oiseaux. Thèse de Géographie, université de Bretagne Occidentale, Brest, 537 p.

Le Guyader D., 2012, Modélisation des activités humaines en mer côtière, Thèse de géographie, Université de Bretagne Occidentale, LETG-Brest Géomer UMR6554, 309 p.

Lloret J., Zaragoza N., Caballero D., Riera V., 2008. Impacts of recreational boating on the marine environment of Cap de Creus (Mediterranean Sea), Ocean and Coastal Management, $\mathrm{n}^{\circ}$ 51, p. 749-754.

Marrou L., 2011 . Nautical frequentation and marina management, Journal of coastal of research, Special issue, $n^{\circ} 61$, p. 126-132.

Мıснот T., 1998. Contribution à la compréhension et à l'analyse des pratiques nautiques sur le Bassin d'Arcachon, tome 1 et 2, Thèse de Doctorat en Sciences et Techniques des Activités Physiques et Sportives, Université de Paris-Sud-Orsay, 610 p.

Мıснот T., 2008. Des territoires marins confrontés aux contraintes du développement durable - Nautisme, côtes bretonnes et développement durable : dynamiques et résistances, in Bessy O. (dir.), Sport, loisir, tourisme et développement durable des territoires, Voiron, Territorial éditions, Presses universitaires du sport, p. 167-176.

Milazzo M., Badalamenti F., Ceccherelli G., Chemello R., 2004. Boat anchoring on Posidonia oceanica beds in 
a marine protected area (Italy, western Mediterranean): effect of anchor types in different anchoring stages, Journal of Experimental Marine Biology and Ecology, vol. 299, $\mathrm{n}^{\circ} 1$, p. 51-62.

Miossec A. (dir.), 2012. Dictionnaire de la mer et des côtes, Rennes, PUR, 550 p.

Nardin G., Berre I., Brigand L., 2008. Un SiG pour connaître et pour gérer la plaisance dans le Finistère, Norois, ${ }^{\circ}$ 206, vol. 1, p. 53-72.

Nautisme en Finistère, 2012. Chiffres clés 2012 du Nautisme en Finistère, 10 p.

Odit France, 2008. Le marché de la plaisance en FranceMieux comprendre les pratiques, les besoins et les attentes des plaisanciers, $210 \mathrm{p}$.

Perras L., 2012. Diagnostic sur le bateau transportable du Pays de Brest. Mémoire de Master 1, Université de Bretagne Occidentale (LETG-Brest Géomer), 137 p.

Peuziat I., Le Berre I. (dir.) 2015. CARTAHU - Cartographie des activités humaines en mer côtière, Expérimentations en Mer d'Iroise, LETG-Brest Géomer, UMR 6554 CNRS, IUEM-UBO, Brest, 87 p.

Peuziat I., 2005. Plaisance et environnement, Pratiques, représentations et impacts de la fréquentation nautique de loisir dans les espaces insulaires, Thèse de doctorat de Géographie, Université de Bretagne Occidentale, Brest, 336 p.

Retière D., 2003. Les bassins de plaisance : structuration et dynamiques d'un territoire, Thèse de doctorat de Géographie, Université de Bretagne Occidentale, Brest, 327 p.

Richez G., 1992. Un port de plaisance sur un littoral deltaïque : le cas de Port-Gardian en Camargue, Méditerranée, vol. 76, $n^{\circ} 3-4$, p. 19-26.
Roux M., 1994. Imaginaire, pratique sportive, valeur de l'espace. La plaisance sur la côte atlantique française, Norois, $n^{\circ} 163$, p. 419-428.

Sidman C., Swell R., Fik T., Fann S., Fann D., Sargent B., 2005. A Recreational Boating Characterization for the Greater Charlotte Harbor, Florida Sea Grant, University of Florida, $94 \mathrm{p}$

SonNic E., 2005. La navigation de plaisance : territoires de pratiques et territoires de gestion en Bretagne, Thèse de doctorat de Géographie, Université de Rennes 2 - Haute-Bretagne, $503 \mathrm{p}$.

Sonnic E., 2004. Le bassin de navigation : d'une pratique de l'espace de loisir à la construction d'un territoire, Norois, $n^{\circ} 190,(2004-1)$, p. 55-66.

Tonini B., Trouillet B., 2005. Les bassins de navigation : éléments d'une typologie. Étude sur la façade atlantique à partir de la réglementation, in Bernard N. (Coord.), Le Nautisme, acteurs, pratiques et territoires, Rennes, Presses universitaires de Rennes, coll. Espace et territoires, 332 p., p. $60-85$.

Trouillet B., 2006. La mer-territoire ou la banalisation de l'espace marin (Golfe de Gascogne). Mappemonde, $\mathrm{n}^{\circ} 84$ (2006-4), 17 p.

Widmer W.M., Underwood A.J., 2004. Factors affecting traffic and anchoring patterns of recreational boats in Sydney Harbour, Australia, Landscape and Urban Planning, vol. 66, $n^{\circ} 3$, p. 173-183. 